\title{
Ruptured Splenic Abscess following Percutaneous Transluminal Angioplasty in a 40-Year-Old Man
}

\author{
C. Rajasekharan T. Jayapal \\ Department of Internal Medicine, Medical College Hospital, Thiruvanthapuram, \\ India
}

\section{Key Words}

Percutaneous transluminal coronary angioplasty $\cdot$ Splenic abscess .

Klebsiella pneumoniae · Peritonitis

\begin{abstract}
The incidence of splenic abscesses is currently $0.14-0.7 \%$ with a reported mortality of $0-47 \%$. The diagnosis of splenic abscess which has ruptured into the abdomen is often overlooked because of its rarity and its misleading clinical presentations. Percutaneous coronary interventions (PCls) and coronary stenting procedures increased from 184,000 to 885,000 (from 335 to 1,550) and from 3,000 to 770,000 (from 5 to 1,350 per one million inhabitants), respectively. A 40 -year-old Asian male presented to our emergency department with upper abdominal pain 5 days after a percutaneous transluminal coronary angioplasty. Clinical examination raised the possibilities of acute pancreatitis and intraabdominal sepsis. An initial ultrasound of the abdomen and blood tests were negative. A computed tomography scan of the abdomen revealed a splenic abscess that had ruptured into the abdomen. Pus culture revealed a multidrug-resistant strain of Klebsiella pneumoniae that was sensitive to meropenem. The patient recovered quickly after open surgical drainage and antibiotic therapy. As this is the second case of splenic abscess and the first case report of a ruptured splenic abscess following a $\mathrm{PCl}$, it will be rational to administer a short course of antibiotic prophylaxis for high-risk immunocompromised patients who are undergoing percutaneous transluminal coronary intervention.
\end{abstract}

\section{Introduction}

The era of interventional cardiology began with the performance of the first successful balloon angioplasty by Andreas Gruentzig in 1977. The data from the 
European Society of Cardiology reveal that the total numbers of coronary angiographies increased from 684,000 in 1992 to 2,238,000 in 2004 (from 1,250 to 3,930 per one million inhabitants). Percutaneous coronary interventions (PCIs) and coronary stenting procedures increased from 184,000 to 885,000 (from 335 to 1,550 ) and from 3,000 to 770,000 (from 5 to 1,350 per one million inhabitants), respectively. These procedures were adopted most aggressively in Germany, with 712,000 angiographies (8,600 per one million inhabitants), 249,000 angioplasties $(3,000$ per one million inhabitants $)$ and 200,000 stenting procedures $(2,400$ per one million inhabitants) in 2004. Data from the New York PCI registry reveal that the rate of mortality following PCI procedure has declined from $0.90 \%$ in 1997 to $0.58 \%$ in 2003 , which represents a reduction of $36 \%(p<0.001)$. Infections following PCIs are extremely rare. Although minor infections have been reported, there are no published cases of splenic abscesses that have ruptured into the abdomen. Splenic abscesses continue to be a rare clinical entity. The diagnosis of fulminant intraabdominal infection which has ruptured into the abdomen is often overlooked because of its rarity and its misleading clinical presentations. Although rare, this case report should be an eye-opener towards ensuring meticulous antiseptic precautions prior to PCIs.

\section{Case Report}

A 40-year-old Asian male was referred from the cardiology unit of another hospital to our emergency department with pain in the left upper abdomen and generalized malaise that had persisted for 3 days. He denied any fever, chest pain, altered bowel habits, nausea or vomiting. He had undergone coronary angioplasty for an inferior wall myocardial infarction in another institution 5 days before presentation and was taking cardiac medications. He had been taking $5 \mathrm{mg}$ of glibenclamide twice daily for 3 years, which poorly controlled his type 2 diabetes mellitus. He had a history of smoking 20 cigarettes per day for the last 7 years and had consumed bootleg liquor daily for more than 7 years.

Upon examination, the patient was conscious and alert with a pulse of $88 \mathrm{bpm}$, a blood pressure of $100 / 70 \mathrm{~mm} \mathrm{Hg}$, a respiratory rate of $18 / \mathrm{min}$ and a temperature of $37^{\circ} \mathrm{C}$. There was no pallor or pedal edema. Physical examination showed tenderness in the epigastrium and left hypochondrium. There was no hepatosplenomegaly. The cardiovascular, respiratory and neurological systems were normal upon examination. Initial laboratory tests included the following: hemoglobin $13.2 \mathrm{~g} / \mathrm{dl}$, total white blood cell count $8,900 / \mathrm{mm}^{3}$ (differential count: neutrophils $82 \%$, lymphocytes $16 \%$, eosinophils $2 \%$ ), erythrocyte sedimentation rate $32 \mathrm{~mm} / \mathrm{h}$, platelet count $185 \times 10^{3} / \mathrm{mm}^{3}$, blood urea nitrogen $32 \mathrm{mg} / \mathrm{dl}$, serum creatinine $0.9 \mathrm{mg} / \mathrm{dl}$, random blood sugar $406 \mathrm{mg} / \mathrm{dl}$, sodium $136 \mathrm{mmol} / \mathrm{l}$, potassium $4.8 \mathrm{mmol} / \mathrm{l}$, serum bilirubin $1.3 \mathrm{mg} / \mathrm{dl}$, direct bilirubin $0.6 \mathrm{mg} / \mathrm{dl}$, serum glutamic oxaloacetic transaminase/glutamic-pyruvic transaminase ratio 84/28 IU, alkaline phosphatase $240 \mathrm{IU}(0-140)$, total protein $6.4 \mathrm{~g} / 100 \mathrm{ml}$, serum albumin $3.1 \mathrm{~g} / 100 \mathrm{ml}$, serum amylase $30 \mathrm{U} / \mathrm{l}$ and serum lipase $10 \mathrm{U} / \mathrm{l}$. The following serologies were reported as negative: Widal test, hepatitis B surface antigen, hepatitis C, Weil's antibody, Brucella agglutination titer, human immunodeficiency virus 1 and 11, antinuclear antibodies, and anti-double-stranded DNA antibody and venereal disease research laboratory test was non-reactive. The patient's serum alpha lipoprotein was $10 \mathrm{ng} / \mathrm{dl}(0-7.2)$ and his serum carcinoembryonic antigen concentration was $2.44 \mathrm{ng} / \mathrm{dl}(0-3.4)$. A 12-lead electrocardiogram showed Q waves and T wave inversion in the inferior leads. Chest X-ray postero-anterior view and plain X-ray of the abdomen in the erect position were normal. Abdominal ultrasound was normal. The patient was prescribed broad-spectrum antibiotics, and his cardiac medications were continued except for aspirin which was withheld temporarily. His blood sugar was controlled with $16 \mathrm{U}$ of regular insulin 3 times daily.

On day 4 of admission, the patient began to experience high, spiking fevers intermittently with chills and rigors. Acetaminophen (650 mg by mouth) provided temporary relief from fever which lasted for up to $6 \mathrm{~h}$. The abdominal pain was increasing in intensity. Thus, the laboratory tests were repeated and reported the following: hemoglobin $13 \mathrm{~g} / \mathrm{dl}$, total white cell count 20,500/mm³ 
(differential count: neutrophils 90\%, lymphocytes 10\%), erythrocyte sedimentation rate $96 \mathrm{~mm} / \mathrm{h}$ and platelet count $140 \times 10^{3} / \mathrm{mm}^{3}$. A peripheral smear showed neutrophilia with a left shift and toxic granules. No abnormal cells were observed. Liver function and renal function tests were normal. Blood culture and urine cultures were determined to be negative. Because of a negative abdominal ultrasound, we proceeded to abdominal computed tomography (CT) scan, which revealed a ruptured splenic abscess (fig. 1) with subcapsular ( $\underline{\text { fig. } 2}$ ) and subdiaphragmatic collection ( $\underline{\text { fig. } 3}$ ). The patient was diagnosed with a ruptured splenic abscess following coronary angioplasty. An emergency laparotomy was performed, and approximately $500 \mathrm{ml}$ of purulent fluid was drained. Culturing and sensitivity of this fluid revealed a multidrug-resistant strain of Klebsiella pneumoniae that was sensitive to meropenem. Therefore, $1 \mathrm{~g}$ of meropenem was administered 3 times daily, and the patient's spleen was salvaged. Antibiotics were continued for 14 days postoperatively. The patient recovered quickly and has been doing well on follow-up.

\section{Discussion}

The total numbers of coronary angiographies, PCIs and coronary stenting procedures increased significantly from 1992 to 2004 [1]. Data from the New York PCI registry reveal that the rate of mortality following PCI has declined from $0.90 \%$ in 1997 (13) to $0.58 \%$ in 2003 (14), which represents a reduction of $36 \%(p<0.001)$ [2]. Current rates of PCI complications remain low. Stathopoulos et al. [3] reported that the overall PCI complication rate was lower during the final 4 years of their study (2003-2006) than in the first 4 years (1999-2002). To explore the reasons for readmission after PCI, Yost et al. [4] analyzed data submitted prospectively to the American College of Cardiology's National Cardiovascular Data Registry on elective, urgent and emergent PCIs performed from January 1, 2007 to April 12, 2010. Of the 4,523 patients who underwent PCIs during the study period, 222 (4.9\%) required readmission within 30 days for the following causes: reasons unrelated to index admission or PCI (41.4\%), cardiac reasons unrelated to PCI (39.2\%), PCI-related complications (13.5\%) and non-cardiac reasons related to the index admission (5.9\%). Infectious complications occurring after percutaneous transluminal coronary angioplasty (PTCA) are extremely rare [5]. In a study of 147 patients undergoing blood culture testing immediately after PCI, $26(17.7 \%)$ had a detectable bacteremia $12 \mathrm{~h}$ after the procedure. Another $12 \%$ of patients had positive blood cultures during the next $12 \mathrm{~h}$ with femoral sheaths still in situ. Coagulase-negative Staphylococcus was the most commonly isolated bacterium. There were no associated clinical sequelae; this finding emphasizes the need for maximum sterility during PCI procedures to avoid infective complications and stent infection [6].

Whereas cardiac catheterization carries a negligible risk of bacteremia, PCI bacteremia occurs frequently (in approximately $30 \%$ of cases); however, clinical sequelae occur rarely in such cases. PCI has a greater bacteremic potential, probably because of the length of the procedure and the repeated insertion of interventional devices into the vascular system [7]. Reports of infectious complications, such as myocardial abscesses directly related to percutaneous transluminal angioplasty, are extremely rare. There have only been a few reported cases of groin infection following femoral artery repuncture for a coronary angioplasty [8]. A case of bacterial pericarditis developed 1 week after coronary angioplasty and stent implantation [5], two cases of septic arthritis of the knee secondary to probable femoral endarteritis, and another with infected hematoma of the groin. Splenic abscesses have not been perceived as common complication after PCI [8]. Alcoholics, diabetics and 
immunosuppressed patients are most susceptible to splenic abscesses [9]. Standard aseptic techniques may be inadequate if early repuncture is performed or if an indwelling line is utilized. Prophylactic antibiotics should be considered in these cases, although their usefulness has not been formally demonstrated so far [10]. The following risk factors may predispose a patient to infectious sequelae following PTCA: early reuse of the initial puncture site, prolonged retention of the femoral sheath, bleeding or hematoma at the femoral sheath insertion site and vascular complications such as pseudoaneurysms [11]. The clinician should be aware of these risks because a patient may develop these potentially serious complications after PTCA [11].

There has been only one report of a splenic abscess following coronary angioplasty [12]. This is the first case report of a splenic abscess that perforated and produced a subdiaphragmatic collection. The proposed mechanism of the splenic abscess in this patient is a splenic infarction caused by dislodged cholesterol emboli during PTCA, followed by a secondary infection that became septic. The evolving infarcts may have been missed in the initial abdominal ultrasound [13]. The absence of fever and the normal laboratory findings at presentation also support this hypothesis. The incidence of splenic abscesses is currently $0.14-0.7 \%[13,14]$, with a reported mortality of $0-47 \%$ [11]. The diagnosis is often overlooked because of its rarity and misleading clinical presentation, as well as the presence of predisposing conditions that obscure its presentation [11]. Even in the most recently published series diagnosis was made only at autopsy in $37 \%$ of cases [12]. In recent years, splenic abscesses have been diagnosed more frequently because of the increasing number of immunocompromised patients and the widespread use of diagnostic imaging modalities, such as CT and ultrasonography. Although Staphylococcus aureus and Streptococcus pyogenes are most frequently reported as the causes of splenic abscesses, several studies have investigated the significant role of Enterobacteriaceae [11,12]. In a study by Chang et al. in Taiwan [15], K. pneumoniae was the most common cause of splenic abscesses $[11,13]$. There are increasing numbers of case reports of fungal and mycobacterial abscesses in the spleen, especially in immunosuppressed patients [16]. These findings show a clear change in the spectrum of infection in recent years. The following five etiological variants can predispose a patient to developing a splenic abscess: trauma, metastatic infection, rheumatologic disorders, contiguous infection and immunosuppression [13].

Abdominal CT is currently the standard for diagnosing a splenic abscess with a sensitivity of $100 \%$ [16]. The characteristic CT findings for splenic abscesses are low-density lesions that fail to enhance with IV contrast. Ultrasound may also yield a diagnosis but is less sensitive [11]. Some recent case series have reported good success rates with CT-guided percutaneous drainage, but the success depends on the radiological findings. This technique can be effective and is superior to splenectomy in selected cases because it preserves the spleen, which has an important role in the immune system [16]. Percutaneous, radiologically guided drainage may be suitable in some cases, but splenectomy with appropriate antibiotics is the definitive treatment [11]. Some recent case series have reported positive outcomes with the non-operative management of splenic abscesses. However, splenectomy is the most reliable way to remove the abscess and may be necessary in more severe cases. Open splenectomy carries a mortality rate of $0-17 \%$ and a risk of postsplenectomy sepsis [11]. Laparoscopic splenectomy may be a safe and effective alternative [11]. 


\section{Conclusions}

Due to advances in interventional cardiology techniques, the number of people undergoing these procedures has increased overwhelmingly. However, the number of drug-resistant organisms is also increasing. There are controversies regarding prophylactic antibiotic therapy in PCI. We strongly advocate antibiotic prophylaxis in patients who are at a high risk of infection or who are immunocompromised. The case described here involved an infection of the spleen, which is a rare site for abscess formation, and further substantiates the importance of antibiotic prophylaxis.

\section{Disclosure Statement}

The authors declare that they have no competing interests. No external funding was obtained.

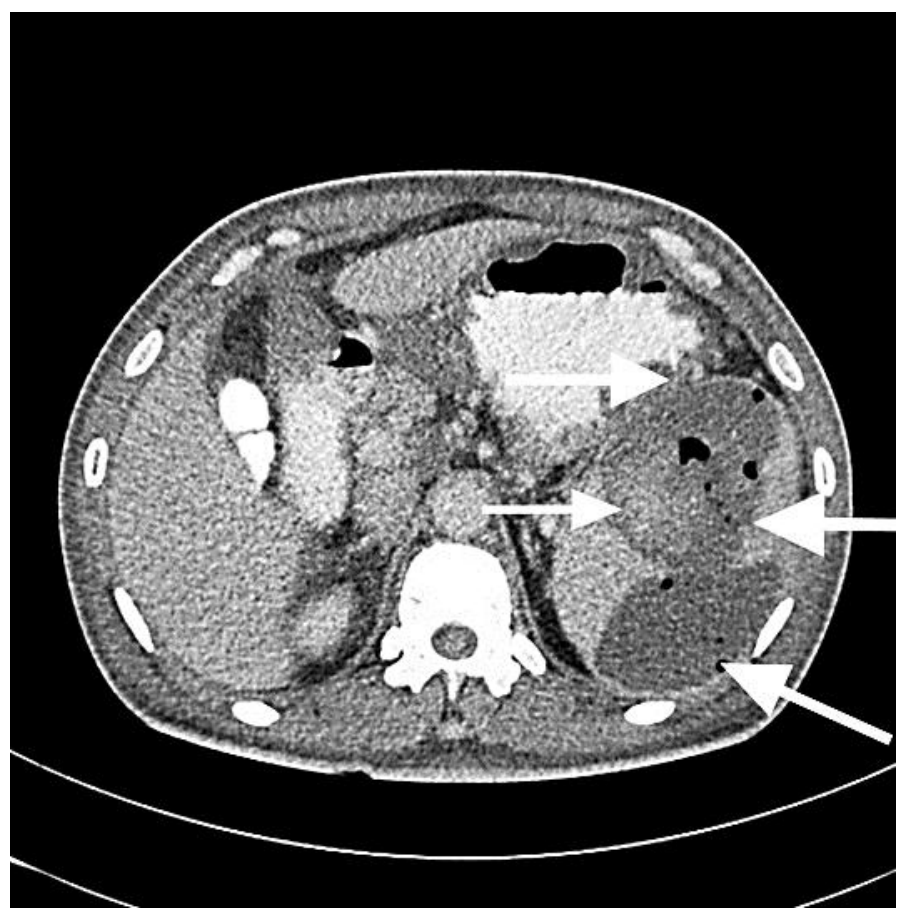

Fig. 1. CT scan of the abdomen showing splenic abscess (white arrows). 


\begin{tabular}{r|l|l|l}
$\begin{array}{r}\text { Case Reports in } \\
\text { Gastroenterology }\end{array}$ & $\begin{array}{l}\text { Case Rep Gastroenterol 2012;6:333-339 } \\
\text { DOI: 10.1159/000339463 }\end{array}$ & $\begin{array}{l}\text { Published online: } \\
\text { May 31, 2012 }\end{array}$ & $\begin{array}{l}\text { @ 2012 S. Karger AG, Basel } \\
\text { ISSN 1662-0631 } \\
\text { www.karger.com/crg }\end{array}$ \\
\hline
\end{tabular}

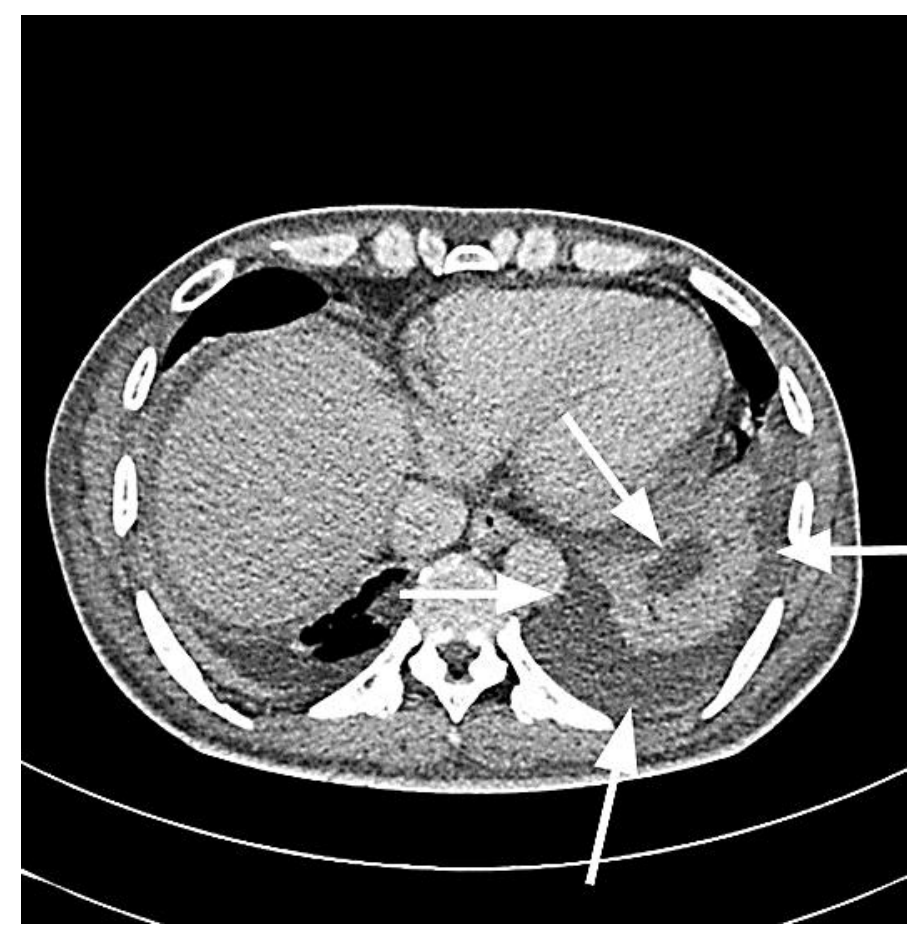

Fig. 2. CT scan of the abdomen showing splenic abscess which has ruptured with subcapsular collection (white arrows).

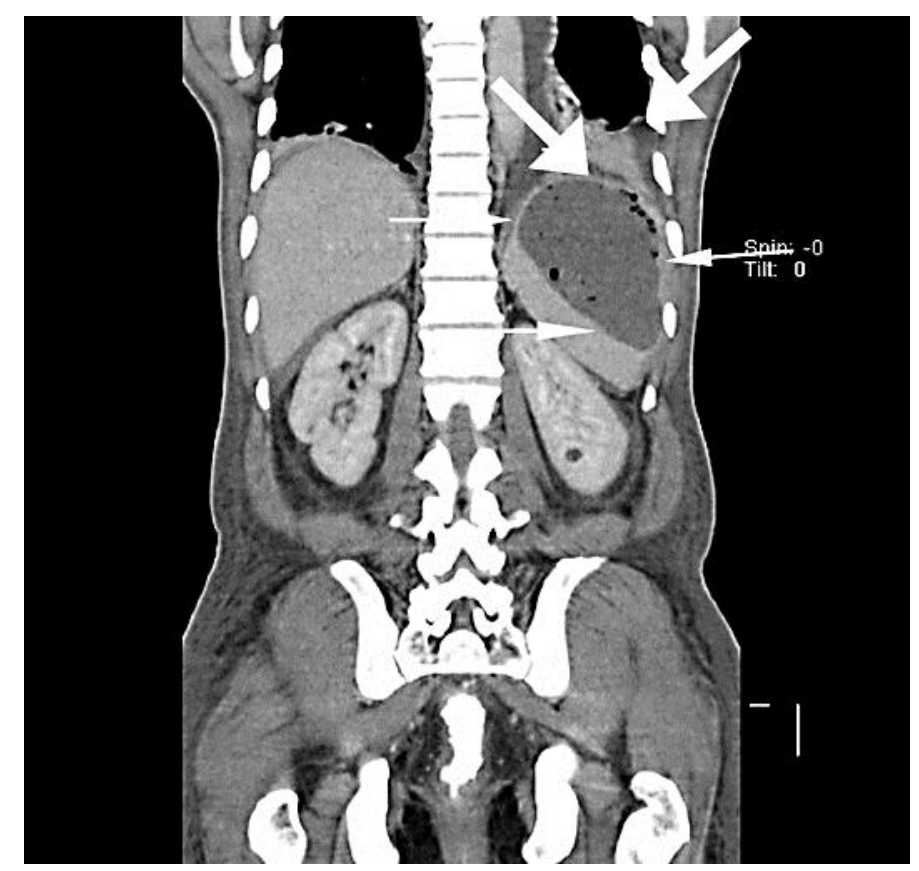

Fig. 3. CT scan of the abdomen showing splenic abscess (thin white arrows) with subdiaphragmatic collection and pleural effusion (thick white arrows). Left side. 


\section{References}

$\checkmark 1$ Cook S, Walker A, Hügli O, Togni M, Meier B: Percutaneous coronary interventions in Europe: prevalence, numerical estimates, and projections based on data up to 2004. Clin Res Cardiol 2007;96:375-382

-2 Resnics FS, Welt FG: The public health hazards of risk avoidance associated with public reporting of risk-adjusted outcomes in coronary intervention. J Am Coll Cardiol 2009;53:825-830.

-3 Stathopoulos I, Jimenez M, Panagopoulos G, et al: The decline in PCI complication rate: 2003-2006 versus 1999-2002. Hellenic J Cardiol 2009;50:379-387.

4 Yost G, et al: The frequency and etiology of readmission in the 30 days after a percutaneous coronary intervention. Baltimore, Society for Cardiovascular Angiography and Interventions, 2011, abstract C-024.

5 Sankari A, Kumar AN, Kabins S, Chandna H, Lieb D: Staphylococcal pericarditis following percutaneous transluminal coronary angioplasty. Catheter Cardiovasc Interv 2000;50:71-73.

6 Ramsdale R, Aziz S, Newall N, Palmer N, Jackson M: Bacteremia following complex percutaneous coronary intervention. J Invas Cardiol 2004;16:632-634.

7 Badshah A, Younas F, Janjua M: Infective mycotic aneurysms presenting as transient acute coronary occlusion and infectious pericarditis. South Med J 2009;102:640-642.

$>8$ Cleveland KO, Gelfand MS: Invasive staphylococcal infections complicating percutaneous transluminal coronary angioplasty. Three cases and review. Clin Infect Dis 1995;21:93-96.

-9 Shim WH, Jang YS, Lee JT, Lee KS: Occult splenic abscess following percutaneous transluminal coronary angioplasty (PTCA): an unrecognized complication of PTCA. Yonsei Med J 1988;29:89-93.

10 Wiener RS, Ong LS: Local infection after percutaneous transluminal coronary angioplasty: relation to early repuncture of ipsilateral femoral artery catheterization and cardiovascular diagnosis. Cathet Cardiovasc Diagn 1989;16:180-181.

11 Lee WS, Choi ST, Kim KK: Splenic abscess: a single institution study and review of the literature. Yonsei Med J 2011;52:288-292.

12 Solomkin JS, Mazuski JE, Bradley JS, et al: Diagnosis and management of complicated intra-abdominal infection in adults and children: guidelines by the Surgical Infection Society and the Infectious Diseases Society of America. Clin Infect Dis 2010;50:133-164.

13 Anderson C, Colvin H: A rare cause of abdominal pain. BMJ 2010;340:c2112.

14 Ulhaci N, Meteoğlu I, Kacar F, Ozbas S: Abscess of the spleen. Pathol Oncol Res 2004;10:234-236.

15 Chang KC, Chuah SK, Changchien CS, et al: Clinical characteristics and prognostic factors of splenic abscess: a review of 67 cases in a single medical center of Taiwan. World J Gastroenterol 2006;12 460-464.

16 Tasar M, Ugurel M: Computed tomography-guided percutaneous drainage of splenic abscess. Clin Imaging 2004;28:44-48. 